\title{
ABOUT A STRESS DEFORMATION CONDITION OF A PIECEWISE-UNIFORM WEDGE WITH A SYSTEM OF COLLINEAR CRACKS AT AN ANTIPLANE DEFORMATION
}

\author{
D. I. BARDZOKAS, S. H. GEVORGYAN, AND S. M. MKHITARYAN
}

Received 13 December 2001

An antiplane problem of a stress deformation condition of a piecewise wedge consisting of two heterogeneous wedges with different opening angles and containing on the line of their attachment a system of arbitrary finite number of collinear cracks is investigated. With the help of Mellin's integral transformation the problem is brought to the solution of the singular integral equation relating to the density of the displacement dislocation on the cracks, which then is reduced to a system of singular integral equations with kernels being represented in the form of sums of Cauchy kernels and regular kernels. This system of equations is solved by the known numerical method. Stress intensity factors (SIF) are calculated and the behavior of characteristic geometric and physical parameters is revealed. Besides, the density of the displacement dislocation on the cracks, their evaluation, and $J$-integrals are calculated.

\section{Introduction}

Numerous problems of antiplane deformation of elastic homogeneous and heterogeneous bodies of different geometric forms, containing cracks, are well known from [3, $8,11,12,13]$. In such problems elastic bodies are usually taken in the form of an infinite space, half-space, layer, infinite circular or noncircular (e.g., elliptic) cylinder spaces with cylindrical opening, and so forth. The problems of antiplane deformation of wedgeshaped bodies with cracks, especially of heterogeneous ones are not sufficiently studied. Here we can mention the scientific works $[4,6]$ and also [15], which is close to the investigation. It should be mentioned here that these problems are of interest in the mechanics of composites and generally in designing various constructions with angular points, which are exposed to force input causing antiplane deformation. Round the angular points there usually occur strong stress concentrations owing to which in bodies there may be formed and propagated cracks.

In the present work on the basis of the theory of elasticity we investigate the problem of a stress deformation condition of a piecewise-uniform wedge-shaped body with an antiplane deformation consisting of two heterogeneous wedges with various opening angles and containing an arbitrary finite number of intercrossing cracks on the seal line. 


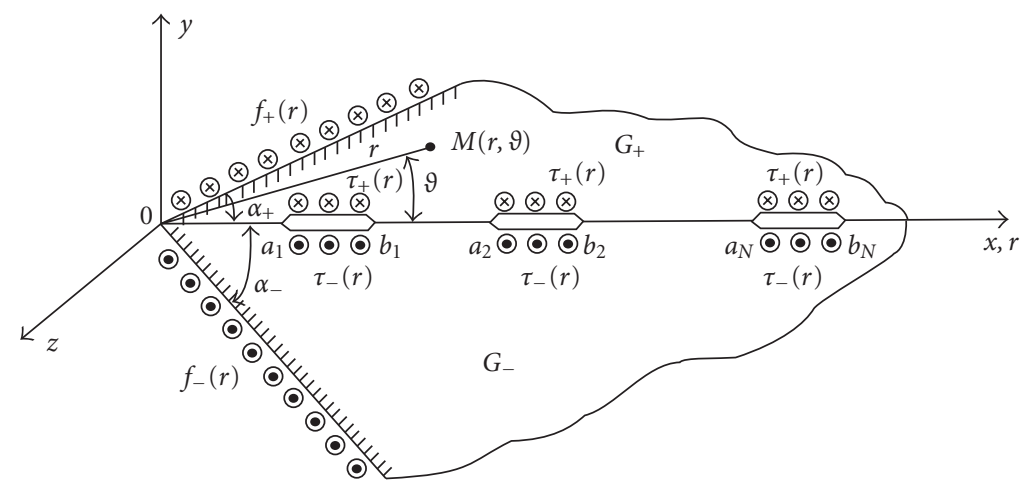

Figure 2.1. Geometry and loading of a piecewise-uniform wedge at an antiplane deformation with a system of collinear internal cracks $L=\bigcup_{k=1}^{N}\left(a_{k}, b_{k}\right)$ at the interface.

With the help of Mellin's integral transformation we derive the principal equation of the problem which on the assembly of the cracks gives the determinative integral equation (DIE) of the problem with respect to the density of the displacement dislocations of the crack edges, but outside of the crack system on the line of their disposition defines the tangential breaking stress. The kernel structure of DIE is investigated and as a result the equation is represented as a sum of its singular parts: Cauchy kernels and regular kernels. Further, DIE is transformed into a system of singular integral equations for the solution of which we use the numerical method developed in $[5,14]$.

After the definition of the density of the displacement dislocations on the cracks from DIE we define the stress intensity factors (SIF), J-integrals, representing the velocities of the energy release of elastic deformation at the end zones of the cracks and opening of the cracks. The behavior of the SIF is studied on a large scale of changes of characteristic geometric and physical parameters.

\section{Formulation of the problem and derivation of determinative equations}

We consider an infinite elastic wedge-shaped body in a right rectangular Cartesian system of coordinates $O x y$, which is in condition of antiplane deformation (longitudinal shear) in the direction of axis $O z$ with datum plane $O x y$ and consists of two heterogeneous wedges with shear moduli $G_{+}, G_{-}$, with opening angles $\alpha_{+}, \alpha_{-}\left(0<\alpha_{+}+\alpha_{-}<2 \pi\right)$, respectively. On the plane of their seal $y=0$ the composite wedge contains a system of the arbitrary number $N$ of nonintercrossing cracks in the form of strips. Traces of these cracks on axis $O x$ form the collection of intervals (Figure 2.1)

$$
L=\bigcup_{k=1}^{N}\left(a_{k}, b_{k}\right) \quad\left(a_{k}<b_{k} ; k=1,2, \ldots, N ; b_{k}<a_{k+1} ; k=1,2, \ldots, N-1\right) \text {. }
$$

Furthermore, let the antiplane body deformation be accomplished by distributing along the wedge faces and crack edges tangential horizontal forces acting in the direction of axis $\mathrm{Oz}$. 
To describe these loads on datum plane $O x y$, we introduce the polar system of coordinates $(r, \theta)$ with a pole at the beginning of the coordinate $O$ and with the polar axis $O r$ coinciding with axis $O x$. Assume that such loads act on the faces of the wedges:

$$
\left.\tau_{\theta z}\right|_{\theta= \pm \alpha_{ \pm}}=f_{ \pm}(r) \quad(0<r<\infty)
$$

and the following loads on the edges of the cracks:

$$
\left.\tau_{\theta z}\right|_{\theta= \pm 0}=-\tau_{ \pm}(r) \quad(r \in L) .
$$

Here the limiting equalities $\theta= \pm 0(r \in L)$ define the upper and lower edges of the crack systems, respectively, $f_{ \pm}(r)$ and $\tau_{ \pm}(r)$ are preliminarily given functions from a rather general class of functions with finite integrals (resultants) in their domains of definition, and $\tau_{\theta z}$ is the stress component.

For these assumptions it is necessary to define the main mechanic characteristics of the given problem, such as the dislocation density, SIF, $J$-integrals, and openings of the cracks.

We derive the determinative equation of the problem. For this purpose the composite wedge must be divided into two parts with the help of the seal line of variable wedges, that is, the polar axis $\theta=0$ :

$$
D_{+}=\left\{0 \leq r<\infty ; 0<\theta<\alpha_{+}\right\}, \quad D_{-}=\left\{0 \leq r<\infty ;-\alpha_{-}<\theta<0\right\},
$$

and here we must introduce the following for consideration of the load:

$$
\left.\tau_{\vartheta z}\right|_{\vartheta= \pm 0}=-T_{ \pm}(r)= \begin{cases}-\tau_{ \pm}(r) & (r \in L), \\ -\tau(r) & \left(r \in L^{\prime}\right)\left(L^{\prime}=[0, \infty) \backslash L\right),\end{cases}
$$

and also the singular different from zero components of the points of displacement of elastic wedges at an antiplane deformation

$$
u_{z}^{ \pm}=w_{ \pm}(r, \theta)\left((r, \theta) \in D_{ \pm}\right)
$$

satisfying Laplace equation in region $D_{ \pm}$. Furthermore taking into account Hooke's law we consider the following boundary problems in region $D_{ \pm}$:

$$
\begin{gathered}
\frac{\partial^{2} w_{ \pm}}{\partial r^{2}}+\frac{1}{r} \frac{\partial w_{ \pm}}{\partial r}+\frac{1}{r^{2}} \frac{\partial^{2} w_{ \pm}}{\partial \theta^{2}}=0 \quad\left((r, \theta) \in D_{ \pm}\right), \\
\left.\tau_{\theta z}\right|_{\theta= \pm 0}=\left.\frac{G_{ \pm}}{r} \frac{\partial w_{ \pm}}{\partial \theta}\right|_{\theta= \pm 0}=-T_{ \pm}(r) \quad(0<r<\infty), \\
\left.\tau_{\theta z}\right|_{\theta= \pm \alpha_{ \pm}}=\left.\frac{G_{ \pm}}{r} \frac{\partial w_{ \pm}}{\partial \theta}\right|_{\theta= \pm \alpha_{ \pm}}=f_{ \pm}(r) \quad(0<r<\infty), \\
\tau_{\theta z}, \tau_{r z} \longrightarrow 0 \quad(r \longrightarrow \infty) .
\end{gathered}
$$


We construct the solution of boundary problems (2.7) by applying the method of Mellin's integral transform. For this purpose we must introduce Mellin's transformations

$$
\begin{aligned}
\bar{w}_{ \pm}(p, \theta) & =\int_{0}^{\infty} w_{ \pm}(r, \theta) r^{p-1} d r, \\
\bar{\tau}_{\theta z}(p, \theta) & =\int_{0}^{\infty} \tau_{\theta z}(r, \theta) r^{p} d r \\
\bar{T}_{ \pm}(p) & =\int_{0}^{\infty} T_{ \pm}(r) r^{p} d r \\
\bar{f}_{ \pm}(p) & =\int_{0}^{\infty} f_{ \pm}(r) r^{p} d r .
\end{aligned}
$$

To define a strip of Mellin's integral of regularities we must assume as in [16] that the stresses $\tau_{\theta z}$ and $\tau_{r z}$ at infinity have the order $1 / r$ and on a wedge face in the vicinity of the tip $(r \rightarrow 0)$ have the order $r^{-\varepsilon}(0<\varepsilon<1)$. Then, to provide the convergence of the integrals $(2.8 \mathrm{~b})$ and $(2.8 \mathrm{~d})$ it is sufficient to assume that the complex variable $p$ changes in limits of the strip

$$
\varepsilon-1<\operatorname{Re} p<0 \quad(0<\varepsilon<1) .
$$

As regards the integrals (2.8a) they should be considered in the limits of the theory of generalized functions. On the whole it is more convenient to consider the Mellin's integral transform applied here as the theory of generalized functions.

Thus, we multiply both sides of the differential equation from (2.7) by $r^{p+1}$, the boundary conditions by $r^{p}$, and then the obtained equalities we integrate for $r$ over the interval $(0, \infty)$. Applying the elementary properties of Mellin's transform we arrive at the following boundary problem for ordinary differential equations:

$$
\begin{gathered}
\frac{d^{2} \bar{w}_{ \pm}}{d \theta^{2}}+p^{2} \bar{w}_{ \pm}=0 \quad\left(\theta \in\left(0, \alpha_{+}\right) \bigcup \theta \in\left(-\alpha_{-}, 0\right)\right), \\
\left.G_{ \pm} \frac{d \bar{w}_{ \pm}}{d \theta}\right|_{\theta= \pm 0}=-\bar{T}_{ \pm}(p),\left.\quad G_{ \pm} \frac{d \bar{w}_{ \pm}}{d \theta}\right|_{\theta= \pm \alpha_{ \pm}}=\bar{f}_{ \pm}(p) .
\end{gathered}
$$

Solutions of the boundary problem (2.10) have the following form:

$$
\begin{aligned}
\bar{w}_{ \pm}(p, \theta)=\mp & \frac{1}{p G_{ \pm}}\left\{\left[\operatorname{ctg}\left(p \alpha_{ \pm}\right) \cos (p \theta) \pm \sin (p \theta)\right] T_{ \pm}(p)+\frac{\cos (p \theta)}{\sin \left(p \alpha_{ \pm}\right)} \bar{f}_{ \pm}(p)\right\} \\
& \left(0 \leq \theta \leq \alpha_{ \pm} ;-\alpha_{-} \leq \theta \leq 0\right),
\end{aligned}
$$

wherefrom we find

$$
\bar{w}_{ \pm}(p, 0)=\mp \frac{1}{p G_{ \pm}}\left[\operatorname{ctg}\left(p \alpha_{ \pm}\right) \bar{T}_{ \pm}(p)+\frac{\bar{f}_{ \pm}(p)}{\sin \left(p \alpha_{ \pm}\right)}\right] .
$$

Hence, by the formula of Mellin's inverse transform we have

$$
\frac{d w_{ \pm}(r, 0)}{d r}= \pm \frac{1}{2 \pi i G_{ \pm}} \int_{\Gamma}\left[\operatorname{ctg}\left(p \alpha_{ \pm}\right) \bar{T}_{ \pm}(p)+\frac{\bar{f}_{ \pm}(p)}{\sin \left(p \alpha_{ \pm}\right)}\right] r^{-p-1} d p
$$


where contour $\Gamma$ is the straight line parallel to the imaginary axis and is located in the strip (2.9). Applying the residue theory or the known integrals from [1, page 301, formula (13) and page 302 , formula (19)] when $n=1$ we easily obtain that

$$
\frac{d w_{ \pm}(r, 0)}{d r}= \pm \frac{r^{\pi / \alpha_{ \pm}-1}}{\alpha_{ \pm} G_{ \pm}}\left[\int_{0}^{\infty} \frac{T_{ \pm}\left(r_{0}\right) d r_{0}}{r_{0}^{\pi / \alpha_{ \pm}}-r^{\pi / \alpha_{ \pm}}}-\int_{0}^{\infty} \frac{f_{ \pm}\left(r_{0}\right) d r_{0}}{r_{0}^{\pi / \alpha_{ \pm}}+r^{\pi / \alpha_{ \pm}}}\right] \quad(0<r<\infty) .
$$

This result in this particular case of the half-planes $\left(\alpha_{+}=\alpha_{-}=\pi\right)$ coincides with the known results of [9].

However, for further investigation it is convenient not to appeal to the original equalities and to manipulate with (2.12) of Mellin's images.

Further, we took into consideration the sum and difference of the derivatives of the displacements on the seal line of heterogeneous wedges:

$$
\begin{aligned}
& \Omega(r)=\frac{d w_{+}(r, 0)}{d r}+\frac{d w_{-}(r, 0)}{d r}, \\
& \Phi(r)=\frac{d w_{+}(r, 0)}{d r}-\frac{d w_{-}(r, 0)}{d r} \quad(0<r<\infty),
\end{aligned}
$$

and also their Mellin's images

$$
\bar{\Omega}(p)=\int_{0}^{\infty} \Omega(r) r^{p} d r, \quad \bar{\Phi}(p)=\int_{0}^{\infty} \Phi(r) r^{p} d r
$$

It is obvious that owing to the infinity of the displacements on $L^{\prime}\left(L^{\prime}=[0, \infty) \backslash L\right.$ is the additional to $L$ system of intervals) we may introduce

$$
\Phi(r)= \begin{cases}\varphi(r) & (r \in L) \\ 0 & \left(r \in L^{\prime}\right)\end{cases}
$$

The function $\varphi(r)$ defines the density of the displacement dislocations on the system of the cracks.

Taking into account the functions introduced by means of (2.12) we get the following system of linear algebraic equations for $\bar{T}_{ \pm}(p)$ :

$$
\begin{aligned}
& \frac{\operatorname{ctg}\left(p \alpha_{+}\right)}{G_{+}} \bar{T}_{+}(p)+\frac{\operatorname{ctg}\left(p \alpha_{-}\right)}{G_{-}} \bar{T}_{-}(p)=\bar{\Phi}(p)-\frac{\bar{f}_{+}(p)}{G_{+} \sin \left(p \alpha_{+}\right)}-\frac{\bar{f}_{-}(p)}{G_{-} \sin \left(p \alpha_{-}\right)} \\
& \frac{\operatorname{ctg}\left(p \alpha_{+}\right)}{G_{+}} \bar{T}_{+}(p)-\frac{\operatorname{ctg}\left(p \alpha_{-}\right)}{G_{-}} \bar{T}_{-}(p)=\bar{\Omega}(p)-\frac{\bar{f}_{+}(p)}{G_{+} \sin \left(p \alpha_{+}\right)}+\frac{\bar{f}_{-}(p)}{G_{-} \sin \left(p \alpha_{-}\right)}
\end{aligned}
$$

The solution of this system has the following form:

$$
\bar{T}_{ \pm}(p)=\frac{G_{ \pm}}{2} \operatorname{tg}\left(p \alpha_{ \pm}\right)[\bar{\Phi}(p) \pm \bar{\Omega}(p)]-\frac{\bar{f}_{ \pm}(p)}{\cos \left(p \alpha_{ \pm}\right)},
$$


250 Stress deformation condition of a piecewise-uniform wedge

hence we form the expression $\bar{T}_{+}(p) \pm \bar{T}_{-}(p)$ as

$$
\begin{aligned}
\bar{T}_{+}(p)+\bar{T}_{-}(p)= & \frac{1}{2}\left[G_{+} \operatorname{tg}\left(p \alpha_{+}\right)+G_{-} \operatorname{tg}\left(p \alpha_{-}\right)\right] \bar{\Phi}(p) \\
& +\frac{1}{2}\left[G_{+} \operatorname{tg}\left(p \alpha_{+}\right)-G_{-} \operatorname{tg}\left(p \alpha_{-}\right)\right] \bar{\Omega}(p) \\
& -\frac{\bar{f}_{+}(p)}{\cos \left(p \alpha_{+}\right)}-\frac{\bar{f}_{-}(p)}{\cos \left(p \alpha_{-}\right)}, \\
\bar{T}_{+}(p)-\bar{T}_{-}(p)= & \frac{1}{2}\left[G_{+} \operatorname{tg}\left(p \alpha_{+}\right)-G_{-} \operatorname{tg}\left(p \alpha_{-}\right)\right] \bar{\Phi}(p) \\
& +\frac{1}{2}\left[G_{+} \operatorname{tg}\left(p \alpha_{+}\right)+G_{-} \operatorname{tg}\left(p \alpha_{-}\right)\right] \bar{\Omega}(p) \\
& -\frac{f_{+}(p)}{\cos \left(p \alpha_{+}\right)}+\frac{f_{-}(p)}{\cos \left(p \alpha_{-}\right)} .
\end{aligned}
$$

Now, from the second equation we define $\bar{\Omega}(p)$ and represent its expression in the first equation. As a result of elementary transformations, we arrive at the following key equation in Mellin's images for our problem:

$$
\begin{aligned}
\bar{T}_{+}(p)+\bar{T}_{-}(p)= & 2 G_{+} \frac{\operatorname{tg}\left(p \alpha_{+}\right) \operatorname{tg}\left(p \alpha_{-}\right)}{\chi \operatorname{tg}\left(p \alpha_{+}\right)+\operatorname{tg}\left(p \alpha_{-}\right)} \bar{\Phi}(p) \\
& +\frac{\chi \operatorname{tg}\left(p \alpha_{+}\right)-\operatorname{tg}\left(p \alpha_{-}\right)}{\chi \operatorname{tg}\left(p \alpha_{+}\right)+\operatorname{tg}\left(p \alpha_{-}\right)}\left[\bar{T}_{+}(p)-\bar{T}_{-}(p)\right] \\
& -\frac{2 \operatorname{tg}\left(p \alpha_{-}\right) \bar{f}_{+}(p)}{\cos \left(p \alpha_{+}\right)\left[\chi \operatorname{tg}\left(p \alpha_{+}\right)+\operatorname{tg}\left(p \alpha_{-}\right)\right]} \\
& -\frac{2 \chi \operatorname{tg}\left(p \alpha_{+}\right) \bar{f}_{-}(p)}{\cos \left(p \alpha_{-}\right)\left[\chi \operatorname{tg}\left(p \alpha_{+}\right)+\operatorname{tg}\left(p \alpha_{-}\right)\right]} \\
& \quad\left(\varepsilon-1<\operatorname{Re} p<0 ; 0<\varepsilon<1, \chi=\frac{G_{+}}{G_{-}}\right) .
\end{aligned}
$$

To write down (2.21) in originals we must mention that the factors introduced into it at $\bar{\Phi}(p), \bar{T}_{+}(p)-\bar{T}_{-}(p)$ and $f_{ \pm}(p)$ are analytical on the imaginary axis of $\operatorname{Re} p=0$ function. Therefore, in the formula of Mellin's inversion integral transform, the imaginary axis may be accepted as the line of integration $\Gamma$, and, hence we can introduce $p=i \lambda(-\infty<\lambda<\infty)$ in (2.21). As a result the key equation (2.21) in originals is represented in the following form:

$$
\begin{aligned}
T_{+}(r)+T_{-}(r)= & -\frac{2 G_{+}}{\pi r} \int_{L} K\left(r, r_{0}\right) \varphi\left(r_{0}\right) d r_{0} \\
& +\frac{1}{\pi r} \int_{L} R\left(r, r_{0}\right)\left[\tau_{+}\left(r_{0}\right)-\tau_{-}\left(r_{0}\right)\right] d r_{0} \\
& -\frac{2}{\pi r} \int_{0}^{\infty} M\left(r, r_{0}\right) f_{+}\left(r_{0}\right) d r_{0}-\frac{2 \chi}{\pi r} \int_{0}^{\infty} N\left(r, r_{0}\right) f_{-}\left(r_{0}\right) d r_{0} \\
& \left(\chi=\frac{G_{+}}{G_{-}} ; 0<r<\infty\right) .
\end{aligned}
$$


The following designations are introduced here:

$$
\begin{aligned}
& K\left(r, r_{0}\right)=\int_{0}^{\infty} \frac{\operatorname{th}\left(\lambda \alpha_{+}\right) \operatorname{th}\left(\lambda \alpha_{-}\right)}{\chi \operatorname{th}\left(\lambda \alpha_{+}\right)+\operatorname{th}\left(\lambda \alpha_{-}\right)} \sin \left(\lambda \ln \frac{r_{0}}{r}\right) d \lambda, \\
& R\left(r, r_{0}\right)=\int_{0}^{\infty} \frac{\chi \operatorname{th}\left(\lambda \alpha_{+}\right)-\operatorname{th}\left(\lambda \alpha_{-}\right)}{\chi \operatorname{th}\left(\lambda \alpha_{+}\right)+\operatorname{th}\left(\lambda \alpha_{-}\right)} \cos \left(\lambda \ln \frac{r_{0}}{r}\right) d \lambda, \\
& M\left(r, r_{0}\right)=\int_{0}^{\infty} \frac{\operatorname{th}\left(\lambda \alpha_{-}\right) \cos \left(\lambda \ln \left(r_{0} / r\right)\right)}{\operatorname{ch}\left(\lambda \alpha_{+}\right)\left[\chi \operatorname{th}\left(\lambda \alpha_{+}\right)+\operatorname{th}\left(\lambda \alpha_{-}\right)\right]} d \lambda, \\
& N\left(r, r_{0}\right)=\int_{0}^{\infty} \frac{\operatorname{th}\left(\lambda \alpha_{+}\right) \cos \left(\lambda \ln \left(r_{0} / r\right)\right)}{\operatorname{ch}\left(\lambda \alpha_{+}\right)\left[\chi \operatorname{th}\left(\lambda \alpha_{+}\right)+\operatorname{th}\left(\lambda \alpha_{-}\right)\right]} d \lambda \quad\left(0<r, r_{0}<\infty\right) .
\end{aligned}
$$

It is obvious that kernels $M\left(r, r_{0}\right)$ and $N\left(r, r_{0}\right)$ are of somewhat quick converging cosineFourier integrals, but kernels $K\left(r, r_{0}\right)$ and $R\left(r, r_{0}\right)$ are diverging, in general sense, sine and cosine-Fourier integrals, which must be understood in the sense of the theory of generalized functions [7]. To investigate the structure of kernels $K\left(r, r_{0}\right)$ and $R\left(r, r_{0}\right)$ we must use the method of asymptotic disintegration of Fourier integrals [7].

Firstly, we consider kernel $K\left(r, r_{0}\right)$ from (2.23a). As

$$
\frac{\operatorname{th}\left(\lambda \alpha_{+}\right) \operatorname{th}\left(\lambda \alpha_{-}\right)}{\chi \operatorname{th}\left(\lambda \alpha_{+}\right)+\operatorname{th}\left(\lambda \alpha_{-}\right)} \sim \frac{1}{\chi+1} \quad(\lambda \longrightarrow+\infty),
$$

with the use of the method of asymptotic analysis of Fourier integrals [7], the kernel will have the form

$$
\begin{aligned}
K\left(r, r_{0}\right)= & \int_{0}^{\infty}\left[\frac{\operatorname{th}\left(\lambda \alpha_{+}\right) \operatorname{th}\left(\lambda \alpha_{-}\right)}{\chi \operatorname{th}\left(\lambda \alpha_{+}\right)+\operatorname{th}\left(\lambda \alpha_{-}\right)}-\frac{1}{\chi+1}\right] \sin \left(\lambda \ln \frac{r_{0}}{r}\right) d \lambda \\
& +\frac{1}{\chi+1} \int_{0}^{\infty} \sin \left(\lambda \ln \frac{r_{0}}{r}\right) d \lambda
\end{aligned}
$$

where the last integral is understood in the sense of the theory of generalized functions and is expressed by the formula [7, page 43]

$$
\int_{0}^{\infty} \sin \left(\lambda \ln \frac{r_{0}}{r}\right) d \lambda=\frac{1}{\ln r_{0}-\ln r}
$$

Taking into account this equation after simple transformations we have

$$
\begin{aligned}
K\left(r, r_{0}\right)= & \frac{1}{\chi+1}\left[\frac{1}{\ln r_{0}-\ln r}-\int_{0}^{\infty} \Omega_{\chi}\left(\lambda, \alpha_{+}, \alpha_{-}\right) \sin \left(\lambda \ln \frac{r_{0}}{r}\right) d \lambda\right] \\
& \left(0<r, r_{0}<\infty\right), \\
\Omega_{\chi}\left(\lambda, \alpha_{+}, \alpha_{-}\right)= & {\left[-\frac{\operatorname{th}\left(\lambda \alpha_{+}\right) \operatorname{th}\left(\lambda \alpha_{-}\right)}{\chi \operatorname{th}\left(\lambda \alpha_{+}\right)+\operatorname{th}\left(\lambda \alpha_{-}\right)}+\frac{1}{\chi+1}\right](\chi+1) } \\
= & \frac{\chi \operatorname{th}\left(\lambda \alpha_{+}\right)\left[1-\operatorname{th}\left(\lambda \alpha_{-}\right)\right]+\operatorname{th}\left(\lambda \alpha_{-}\right)\left[1-\operatorname{th}\left(\lambda \alpha_{+}\right)\right]}{\chi \operatorname{th}\left(\lambda \alpha_{+}\right)+\operatorname{th}\left(\lambda \alpha_{-}\right)} .
\end{aligned}
$$


252 Stress deformation condition of a piecewise-uniform wedge

Further, we represent function th $(\lambda \alpha)(\lambda, \alpha>0)$ as absolutely converging infinite series

$$
\operatorname{th}(\lambda \alpha)=1+2 \sum_{k=1}^{\infty}(-1)^{k} e^{-2 k \lambda \alpha} \quad(0<\lambda, \alpha<\infty),
$$

with the help of which for function (2.27b) we may write down the following asymptotic formulas:

$$
\begin{aligned}
\Omega_{\chi}\left(\lambda, \alpha_{+}, \alpha_{-}\right) \sim 2 \psi_{K}\left(\chi, \alpha_{+}, \alpha_{-}\right) e^{-2 \gamma \lambda}+o\left(e^{-2 \gamma \lambda}\right) & (\lambda \longrightarrow+\infty), \\
\gamma=\min \left(\alpha_{+}, \alpha_{-}\right) & \\
\psi_{K}\left(\chi, \alpha_{+}, \alpha_{-}\right) & = \begin{cases}(\chi+1)^{-1} & \left(\alpha_{+}<\alpha_{-}\right) \\
1 & \left(\alpha_{+}=\alpha_{-}\right) \\
\chi(\chi+1)^{-1} & \left(\alpha_{+}>\alpha_{-}\right) .\end{cases}
\end{aligned}
$$

Taking into account the expression of the known sine-Fourier integral from the exponential function in [1, page 71 , formula $2.4(1)]$ with the help of $(2.29 a)$, we obtain the following representation of kernel (2.27a):

$$
\begin{aligned}
& K\left(r, r_{0}\right)= \frac{1}{\chi+1}\left[\frac{1}{\ln r_{0}-\ln r}-K_{0}\left(r, r_{0}\right)\right], \\
& K_{0}\left(r, r_{0}\right)= 2 \psi_{K}\left(\chi, \alpha_{+}, \alpha_{-}\right) \frac{\ln r_{0}-\ln r}{\left(\ln r_{0}-\ln r\right)^{2}+4 \gamma^{2}}+Q_{0}\left(r, r_{0}\right), \\
& Q_{0}\left(r, r_{0}\right)=\int_{0}^{\infty}\left[\Omega_{\chi}\left(\lambda, \alpha_{+}, \alpha_{-}\right)-2 \psi_{K}\left(\chi, \alpha_{+}, \alpha_{-}\right) e^{-2 \gamma \lambda}\right] \\
& \times \sin \left(\lambda \ln \frac{r_{0}}{r}\right) d \lambda \quad\left(0<r, r_{0}<\infty\right),
\end{aligned}
$$

where $\gamma$ and functions $\Omega_{\chi}\left(\lambda, \alpha_{+}, \alpha_{-}\right), \psi_{K}\left(x, \alpha_{+}, \alpha_{-}\right)$are given by the formulas $(2.29 \mathrm{~b})$, $(2.27 \mathrm{~b})$, and $(2.29 \mathrm{c})$, respectively. In formula (2.30a) kernel $K\left(r, r_{0}\right)$ is represented by the sum of the principal and regular parts, and regular kernel $Q_{0}\left(r, r_{0}\right)$ from $(2.30 c)$, according to $(2.29 \mathrm{a})$, is somewhat quickly converging sine-Fourier integral.

Passing to the investigation of the structure of kernel $R\left(r, r_{0}\right)$ from $(2.23 \mathrm{~b})$, we find that

$$
\frac{\chi \operatorname{th}\left(\lambda \alpha_{+}\right)-\operatorname{th}\left(\lambda \alpha_{-}\right)}{\chi \operatorname{th}\left(\lambda \alpha_{+}\right)+\operatorname{th}\left(\lambda \alpha_{-}\right)} \sim \frac{\chi-1}{\chi+1} \quad(\lambda \longrightarrow+\infty) .
$$

Hence we may write

$$
\begin{aligned}
R\left(r, r_{0}\right)= & \int_{0}^{\infty}\left[\frac{\chi \operatorname{th}\left(\lambda \alpha_{+}\right)-\operatorname{th}\left(\lambda \alpha_{-}\right)}{\chi \operatorname{th}\left(\lambda \alpha_{+}\right)+\operatorname{th}\left(\lambda \alpha_{-}\right)}-\frac{\chi-1}{\chi+1}\right] \cos \left(\lambda \ln \frac{r_{0}}{r}\right) d \lambda \\
& +\frac{\chi-1}{\chi+1} \int_{0}^{\infty} \cos \left(\lambda \ln \frac{r_{0}}{r}\right) d \lambda \quad\left(0<r, r_{0}<\infty\right),
\end{aligned}
$$


where the last integral is again understood in the sense of the theory of generalized functions. As in [7, page 43],

$$
\int_{0}^{\infty} \cos \lambda x d \lambda=\pi \delta(x)
$$

where $\delta(x)$ is known as Dirac's delta function, after simple transformations we have

$$
\begin{aligned}
& R\left(r, r_{0}\right)=\pi \frac{\chi-1}{\chi+1} \delta\left(\ln \frac{r_{0}}{r}\right)+\frac{2 \chi}{1+\chi} R_{0}\left(r, r_{0}\right) \quad\left(0<r, r_{0}<\infty\right), \\
& R_{0}\left(r, r_{0}\right)=\int_{0}^{\infty} \frac{\operatorname{th}\left(\lambda \alpha_{+}\right)-\operatorname{th}\left(\lambda \alpha_{-}\right)}{\chi \operatorname{th}\left(\lambda \alpha_{+}\right)+\operatorname{th}\left(\lambda \alpha_{-}\right)} \cos \left(\lambda, \ln \frac{r_{0}}{r}\right) d \lambda .
\end{aligned}
$$

Kernel $R_{0}\left(r, r_{0}\right)$ is the ordinary converging cosine-Fourier integral by the same consideration as above. Taking into account the expression of the known cosine-Fourier integral from the exponential function in [1, page 23, formula $1.4(1)]$ we obtain

$$
\begin{aligned}
R_{0}\left(r, r_{0}\right) & =-4 \psi_{R}\left(\chi, \alpha_{+}, \alpha_{-}\right) \frac{\gamma}{\left(\ln r_{0}-\ln r\right)^{2}+4 \gamma^{2}}+P_{0}\left(r, r_{0}\right) \quad\left(0<r, r_{0}<\infty\right), \\
P_{0}\left(r, r_{0}\right) & =\int_{0}^{\infty}\left[\frac{\operatorname{th}\left(\lambda \alpha_{+}\right)-\operatorname{th}\left(\lambda \alpha_{-}\right)}{\chi \operatorname{th}\left(\lambda \alpha_{+}\right)+\operatorname{th}\left(\lambda \alpha_{-}\right)}+2 \psi_{R}\left(x, \alpha_{+}, \alpha_{-}\right) e^{-2 \gamma \lambda}\right] \cos \left(\lambda \ln \frac{r_{0}}{r}\right) d \lambda, \\
\psi_{R}\left(\chi, \alpha_{+}, \alpha_{-}\right) & = \begin{cases}(\chi+1)^{-1} & \left(\alpha_{+}<\alpha_{-}\right), \\
0 & \left(\alpha_{+}=\alpha_{-}\right), \\
-(\chi+1)^{-1} & \left(\alpha_{+}>\alpha_{-}\right) .\end{cases}
\end{aligned}
$$

Kernels $P_{0}\left(r, r_{0}\right)$ are somewhat quickly converging cosine-Fourier integral.

Then taking into account (2.30a), (2.30b), and (2.34) we finally write the key equation of the problem (2.22) as follows:

$$
\begin{aligned}
T_{+}(r)+\chi T_{-}(r)= & -\frac{G_{+}}{\pi r} \int_{L}\left[\frac{1}{\ln r_{0}-\ln r}-K_{0}\left(r, r_{0}\right)\right] \varphi\left(r_{0}\right) d r_{0} \\
& +\frac{\chi}{\pi r} \int_{L} R_{0}\left(r, r_{0}\right)\left[\tau_{+}\left(r_{0}\right)-\tau_{-}\left(r_{0}\right)\right] d r_{0} \\
& -\frac{1+\chi}{\pi r} \int_{0}^{\infty} M\left(r, r_{0}\right) f_{+}\left(r_{0}\right) d r_{0} \\
& -\frac{\chi(1+\chi)}{\pi r} \int_{0}^{\infty} N\left(r, r_{0}\right) f_{-}\left(r_{0}\right) d r_{0} \quad\left(0<r<\infty ; \chi=\frac{G_{+}}{G_{-}}\right) .
\end{aligned}
$$

Here kernels $K_{0}\left(r, r_{0}\right), R_{0}\left(r, r_{0}\right), M\left(r, r_{0}\right)$, and $N\left(r, r_{0}\right)$ are given by formulas $(2.30 \mathrm{~b})$, (2.30c), (2.34b), (2.23c), and (2.23d), respectively, and for kernel $R_{0}\left(r, r_{0}\right)$ the representation $(2.35)$ is valid. 
Further, we must consider the key equation (2.36) on the system of cracks $L$. As a result we arrive at the following determinative integral equation (DIE) of the problem with respect to the displacement dislocation on $\operatorname{crack} \varphi(r)$ :

$$
\begin{gathered}
\frac{1}{\pi} \int_{L}\left[\frac{1}{\ln r_{0}-\ln r}-K_{0}\left(r, r_{0}\right)\right] \varphi\left(r_{0}\right) d r_{0}=F(r) \quad(r \in L), \\
F(r)=-\frac{\tau_{+}(r)+\chi \tau_{-}(r)}{G_{+}} r+\frac{\chi}{\pi G_{+}} \int_{L} R_{0}\left(r, r_{0}\right)\left[\tau_{+}\left(r_{0}\right)-\tau_{-}\left(r_{0}\right)\right] d r_{0} \\
-\frac{1+\chi}{\pi G_{+}} \int_{0}^{\infty} M\left(r, r_{0}\right) f_{+}\left(r_{0}\right) d r_{0}-\frac{\chi(1+\chi)}{\pi G_{+}} \int_{0}^{\infty} N\left(r, r_{0}\right) f_{-}\left(r_{0}\right) d r_{0} \quad(r \in L) .
\end{gathered}
$$

The solution of DIE (2.37a) must satisfy the specific conditions. Without discussion of various particular cases of the characteristic parameters $\alpha_{+}, \alpha_{-}, x$ and the case of a crack reaching the tip of a composite wedge, where DIE (2.37a) admits closed solutions and which is the subject of a special investigation, we must consider only internal nonboundary end cracks, that is, we must assume that $a_{1}>0$ and the sum of interval lengths of system $L$ is finite (Figure 2.1). Then these conditions will be

$$
\int_{a_{k}}^{b_{k}} \varphi\left(r_{0}\right) d r_{0}=0 \quad(k=\overline{1, N})
$$

expressing the conditions of unambiguity (continuity) of the displacements at the tips of the cracks.

Hence, the key equation (2.36) must be considered apart from the system of cracks on the line of their location, that is, on $L^{\prime}$. As a result we obtain the following expression of the tangential breaking stresses:

$$
\begin{aligned}
-\tau_{\theta z}(r, 0)=\tau(r)= & -\frac{G_{+}}{\pi(1+\chi) r} \int_{L}\left[\frac{1}{\ln r_{0}-\ln r}-K\left(r, r_{0}\right)\right] \varphi\left(r_{0}\right) d r_{0} \\
& +\frac{\chi}{\pi(1+\chi) r} \int_{L} R_{0}\left(r, r_{0}\right)\left[\tau_{+}\left(r_{0}\right)-\tau_{-}\left(r_{0}\right)\right] d r_{0} \\
& -\frac{1}{\pi r} \int_{0}^{\infty} M\left(r, r_{0}\right) f_{+}\left(r_{0}\right) d r_{0} \\
& -\frac{\chi}{\pi r} \int_{0}^{\infty} N\left(r, r_{0}\right) f_{-}\left(r_{0}\right) d r_{0} \quad\left(r \in L^{\prime}\right) .
\end{aligned}
$$

Thus, after constructing the solution of DIE (2.37a) under the conditions of (2.38) the tangential breaking stress is determined by formula (2.39).

\section{Reduction of DIE to a system of singular integral equations and description of the numerical method of its solution}

For this purpose, firstly, we appeal to the new variables in (2.37)

$$
r=a_{1} e^{t}, \quad r_{0}=a_{1} e^{u}
$$


where we assume $a_{1}>0$. Then DIE (2.37a) is transformed into the following singular integral equations:

$$
\begin{gathered}
\frac{1}{\pi} \int_{L_{0}}\left[\frac{1}{u-t}-\widetilde{K}_{0}(u-t)\right] \varphi_{0}(u) d u=F_{0}(t) \quad\left(t \in L_{0}\right), \\
L_{0}=\bigcup_{k=1}^{N}\left(\alpha_{k}, \beta_{k}\right), \\
\alpha_{k}=\ln \left(\frac{a_{k}}{a_{1}}\right), \quad \beta_{k}=\ln \left(\frac{b_{k}}{a_{1}}\right), \\
\left(\alpha_{1}=0, \alpha_{k}>0, k=\overline{2, N} ; \beta_{k}>0, k=\overline{1, N}\right) .
\end{gathered}
$$

Here we introduce the following designations:

$$
\begin{aligned}
\varphi_{0}(t)= & \frac{r \varphi(r)}{a_{1}}=e^{t} \varphi\left(a_{1} e^{t}\right) \\
\widetilde{K}_{0}(u-t)= & K_{0}\left(r, r_{0}\right)=K_{0}\left(a_{1} e^{t}, a_{1} e^{u}\right) \\
= & 2 \psi_{K}\left(\chi, \alpha_{+}, \alpha_{-}\right) \frac{u-t}{(u-t)^{2}+4 \gamma^{2}}+\widetilde{Q}_{0}(u-t) \\
\widetilde{Q}_{0}(u-t)= & Q_{0}\left(r, r_{0}\right)=Q_{0}\left(a_{1} e^{t}, a_{1} e^{u}\right) \\
F_{0}(t)= & F(r)=F\left(a_{1} e^{t}\right)=-\left[\tau_{+}^{0}(t)+\chi \tau_{-}^{0}(t)\right]+\frac{\chi}{\pi} \int_{L_{0}} \widetilde{R}_{0}(u-t)\left[\tau_{+}^{0}(u)-\tau_{-}^{0}(u)\right] d u \\
& -\frac{1+\chi}{\pi} \int_{-\infty}^{\infty} \widetilde{M}_{0}(u-t) f_{+}^{0}(u) d u-\frac{\chi(1+\chi)}{\pi} \int_{-\infty}^{\infty} \widetilde{N}_{0}(u-t) f_{-}^{0}(u) d u \\
\tau_{ \pm}^{0}(t)= & \frac{r \tau_{ \pm}(r)}{G_{+} a_{1}}=\frac{e^{t} \tau_{ \pm}\left(a_{1} e^{t}\right)}{G_{+}}, \\
f_{ \pm}^{0}(t)= & \frac{r f_{ \pm}(r)}{G_{+} a_{1}}=\frac{e^{t} f_{ \pm}\left(a_{1} e^{t}\right)}{G_{+}}, \\
\widetilde{R}_{0}(u-t)= & R_{0}\left(r, r_{0}\right)=R_{0}\left(a_{1} e^{t}, a_{1} e^{u}\right), \\
\widetilde{M}_{0}(u-t)= & M\left(r, r_{0}\right)=M\left(a_{1} e^{t}, a_{1} e^{u}\right), \\
\tilde{N}_{0}(u-t)= & N\left(r, r_{0}\right)=N\left(a_{1} e^{t}, a_{1} e^{u}\right) .
\end{aligned}
$$

It should be mentioned here that the kernel of (3.2a) is presented by the algebraic sum of its singular part as Cauchy kernel $1 /(u-t)$ and in the regular part as a regular kernel from the difference of arguments $\widetilde{K}_{0}(u-t)$ expressed by formulas (3.3b), (2.30b), (2.30c), and (2.27b).

Now, we transform each interval $\left(\alpha_{k}, \beta_{k}\right)$ of the collection $L_{0}$ from (3.2b) into the interval $(-1,1)$, for which in $(3.2 \mathrm{a})$ we introduce

$$
t=\frac{\beta_{k}-\alpha_{k}}{2} \xi+\frac{\beta_{k}+\alpha_{k}}{2}, \quad u=\frac{\beta_{k}-\alpha_{k}}{2} \eta+\frac{\beta_{k}+\alpha_{k}}{2} \quad(k=\overline{1, N},-1<\xi, \eta<1) .
$$


After elementary transformations we reduce (3.2a) into the following system of the singular integral equations:

$$
\begin{gathered}
\frac{1}{\pi} \int_{-1}^{1}\left\{\frac{1}{\eta-\xi}-\nu_{k} \widetilde{Q}_{0}\left[\nu_{k}(\eta-\xi)\right]-\frac{2(\eta-\xi) \psi_{K}\left(\chi, \alpha_{+}, \alpha_{-}\right)}{(\eta-\xi)^{2}+16 \gamma_{k}^{2}}\right\} \varphi_{k}^{0}(\eta) d \eta \\
+\frac{1}{\pi} \sum_{\substack{m=1 \\
m \neq k}}^{N} \int_{-1}^{1}\left\{\frac{1}{\eta-\delta_{k m} \xi}-\nu_{m} \widetilde{Q}_{0}\left[\nu_{m}\left(\eta-\delta_{k m} \xi\right)\right]-\frac{2\left(\eta-\delta_{k m} \xi\right) \psi_{K}\left(\chi, \alpha_{+}, \alpha_{-}\right)}{\left(\eta-\delta_{k m} \xi\right)^{2}+16 \gamma_{m}^{2}}\right\} \\
\times \varphi_{m}^{0}(\eta) d \eta=F_{k}^{0}(\xi) \quad(-1<\xi<1 ; k=\overline{1, N}) \\
\varphi_{m}^{0}(\xi)=\varphi_{0}\left(\frac{\beta_{m}-\alpha_{m}}{2} \xi+\frac{\beta_{m}+\alpha_{m}}{2}\right) \\
F_{k}^{0}(\xi)=F_{0}\left(\frac{\beta_{k}-\alpha_{k}}{2} \xi+\frac{\beta_{k}+\alpha_{k}}{2}\right) \quad(k, m=\overline{1, N}) \\
\gamma_{m}=\frac{\nu_{m}}{\beta_{m}-\alpha_{m}} \quad \frac{\left(\gamma=\min \left(\alpha_{+}, \alpha_{-}\right)\right)}{2}, \\
\delta_{k m}=\frac{\beta_{k}-\alpha_{k}}{\beta_{m}-\alpha_{m}}
\end{gathered}
$$

Here the designations $\widetilde{Q}_{0}(u-t), \varphi_{0}(t)$, and $F_{0}(t)$ are the same as in (3.3b) and (3.3i). With the help of the same formulas of substitution of the variables (3.1) and (3.4), the conditions of (2.38) are transformed into the following:

$$
\int_{-1}^{1} \varphi_{k}^{0}(\eta) d \eta=0 \quad(k=\overline{1, N}) .
$$

Thus DIE (2.37a) may be reduced to the system of singular integral equations (3.5a) under conditions (3.6).

Here for system (3.5) we can apply the known numerical method of the solution of singular integral equations $[5,14]$ based on Gauss quadrature formula for a singular integral of Cauchy type and for an ordinary integral containing positive weighting functions. This method is stated briefly in [12] for problems of cracks. Following [12] we assume the natural number $M$ and introduce the roots of Chebyshev's polynomial of the first type $T_{M}(\eta)=\cos (M \arccos \eta):$

$$
\eta_{\ell}=\cos \left(\frac{2 \ell-1}{2 M} \pi\right) \quad(\ell=\overline{1, M}),
$$

which are the nodes of the mentioned quadrature formulas. We must also introduce the roots of Chebyshev's polynomial of the second type $U_{M-1}(\xi)=\sin (M \arccos \xi) / \sqrt{1-\xi^{2}}$, determined by the formula

$$
\xi_{n}=\cos \frac{\pi n}{M} \quad(n=\overline{1, M-1})
$$


Then, we represent the solution of system (3.5a) in the form

$$
\varphi_{k}^{0}(\xi)=\frac{X_{k}^{0}(\xi, \chi)}{\sqrt{1-\xi^{2}}} \quad(-1<\xi<1 ; k=\overline{1, N})
$$

where function $X_{k}^{0}(\xi, \chi)\left(-1 \leq \xi, \chi=G_{+} / G_{-}\right)$belongs to Hölder's class of functions. Proceeding from (3.9) and (3.7)-(3.8) and according to the given method and conditions (3.6) we must reduce the system (3.5a) to the following finite system of linear algebraic equations:

$$
\begin{aligned}
& \sum_{\ell=1}^{M} \frac{X_{k}^{0}(\eta, \chi)}{M}\left\{\frac{1}{\eta_{\ell}-\xi_{n}}-v_{k} \widetilde{Q}_{0}\left[\nu_{k}\left(\eta_{\ell}-\xi_{n}\right)\right]-\frac{2\left(\eta_{\ell}-\xi_{n}\right) \psi_{K}\left(\chi, \alpha_{+}, \alpha_{-}\right)}{\left(\eta_{\ell}-\xi_{n}\right)^{2}+16 \gamma_{k}^{2}}\right\} \\
& +\sum_{\ell=1}^{M} \sum_{\substack{m=1 \\
m \neq k}}^{N} \frac{X_{m}^{0}\left(\eta_{\ell}, \chi\right)}{M}\left\{\frac{1}{\eta_{\ell}-\delta_{k m} \xi_{n}}-\nu_{m} \widetilde{Q}_{0}\left[\nu_{m}\left(\eta_{\ell}-\delta_{k m} \xi_{n}\right)\right]\right. \\
& \left.-\frac{2\left(\eta_{\ell}-\delta_{k m} \xi_{n}\right) \psi_{K}\left(\chi, \alpha_{+}, \alpha_{-}\right)}{\left(\eta_{\ell}-\delta_{k m} \xi_{n}\right)^{2}+16 \gamma_{m}^{2}}\right\}=F_{k}^{0}\left(\xi_{n}\right) \\
& (n=\overline{1, M-1} ; k=\overline{1, N}) \text {, } \\
& \sum_{\ell=1}^{N} X_{k}^{0}\left(\eta_{\ell}, \chi\right)=0 \text {. }
\end{aligned}
$$

Let $M N$ denote the number of unknowns $X_{k}^{0}\left(\eta_{\ell}, \chi\right)$ in system (3.10), and also the number of the equations.

\section{Expressions of principal mechanic characteristics of the problem}

By solution of the algebraic system (3.10) we express basic mechanic characteristics of the stated problem of cracks, which are the stress intensity factors (SIF), J-integrals, and openings of the cracks.

Firstly, we solve SIF. As in case of a homogeneous body with antiplane deformation we determine the SIF at the crack tips $\left(a_{k}, b_{k}\right)$ by formulas

$$
\begin{aligned}
& K_{I I I}\left(a_{k}\right)=\lim _{r \rightarrow a_{k}-0}\left[\sqrt{2 \pi\left(a_{k}-r\right)} \tau_{\theta z}\right]=-\lim _{r \rightarrow a_{k}-0}\left[\sqrt{2 \pi\left(a_{k}-r\right)} \tau(r)\right], \\
& K_{I I I}\left(b_{k}\right)=\lim _{r \rightarrow b_{k}+0}\left[\sqrt{2 \pi\left(r-b_{k}\right)} \tau_{\theta z}\right]=-\lim _{r \rightarrow b_{k}+0}\left[\sqrt{2 \pi\left(r-b_{k}\right)} \tau(r)\right] \quad(k=\overline{1, N),}
\end{aligned}
$$

where $\tau(r)$ is expressed by the formula (2.39). With the help of (2.39) the formula (4.1) obtains the form

$$
K_{I I I}\left(a_{k}\right)=\frac{\sqrt{2 \pi} G_{+}}{\pi(1+\chi) a_{k}} \lim _{r \rightarrow a_{k}-0}\left[\sqrt{a_{k}-r} \int_{a_{k}}^{b_{k}} \frac{\varphi\left(r_{0}\right)}{\ln r_{0}-\ln r} d r_{0}\right] .
$$


258 Stress deformation condition of a piecewise-uniform wedge

Substitution of variables (3.1) in this equality gives

$$
K_{I I I}\left(a_{k}\right)=\frac{\sqrt{2 \pi} a_{1} G_{+}}{\pi(1+\chi) \sqrt{a_{k}}} \lim _{t \rightarrow a_{k}-0}\left[\sqrt{a_{k}-t} \int_{\alpha_{k}}^{\beta_{k}} \frac{\varphi_{0}(u)}{u-t} d u\right] .
$$

Now, the density Cauchy integral in the vicinity of the point $\alpha_{k}$ can be represented as

$$
\varphi_{0}(t)=\frac{\varphi_{0}^{*}(t)}{\sqrt{t-\alpha_{k}}} \quad\left(t \longrightarrow \alpha_{k}+0\right)
$$

where $\varphi_{0}^{*}(t)$ is Hölder's function and we can take the advantage of known properties of the Cauchy type integral at the end points of integration intervals [10]. As a result we obtain

$$
K_{I I I}\left(a_{k}\right)=\frac{\sqrt{2 \pi} a_{1} G_{+}}{(1+\chi) \sqrt{a_{k}}} \lim _{t \rightarrow \alpha_{k}+0}\left[\sqrt{t-\alpha_{k}} \varphi_{0}(t)\right] .
$$

Going back to the former variable $r$ according to (3.1) we finally find

$$
K_{I I I}\left(a_{k}\right)=\frac{G_{+}}{1+\chi} \lim _{r \rightarrow a_{k}+0}\left[\sqrt{2 \pi\left(r-a_{k}\right)} \varphi(r)\right] \quad(k=\overline{1, N}) .
$$

In the same way formula (4.1b) is transformed as follows:

$$
K_{I I I}\left(b_{k}\right)=-\frac{G_{+}}{1+\chi} \lim _{r \rightarrow b_{k}-0}\left[\sqrt{2 \pi\left(b_{k}-r\right)} \varphi(r)\right] \quad(k=\overline{1, N}) .
$$

It should be mentioned here that if in formulas for SIF in case of homogeneous body $[11,13]$

$$
K_{I I I}\left(\begin{array}{l}
a_{k} \\
b_{k}
\end{array}\right)= \pm \frac{G}{2} \lim _{\substack{r \rightarrow a_{k}+0 \\
r \rightarrow b_{k}-0}}\left[\sqrt{2 \pi\left|\begin{array}{l}
a_{k} \\
b_{k}
\end{array}-r\right|} \mid \varphi(r)\right]
$$

we substitute the shear modulus $G$ by the given shear modulus $G_{0}$

$$
G_{0}=\frac{2 G_{+} G_{-}}{G_{+}+G_{-}}=\frac{2 G_{+}}{1+\chi} \quad\left(\chi=\frac{G_{+}}{G_{-}}\right)
$$

that is, if a piecewise-uniform body with the elastic constants $G_{+}, G_{-}$is substituted by a homogeneous body with the elastic constant $G_{0}$, they will go to formulas (4.6) and (4.7). Hence, substitution of $G$ by $G_{0}$ in the given sense at the antiplane deformation creates some correspondence between piecewise-uniform and homogeneous bodies with cracks. Formulas (4.6)-(4.7) are analogous to known formulas of the plane theory of elasticity for homogeneous bodies with cracks [2]. 
Now, we express SIF as functions $X_{k}^{0}(\xi, \chi)$ from (3.9). For this purpose in (4.6)-(4.7) we consequently move to new variables (3.1) and (3.4), and, then, we allow for (3.2c) and (3.2d). After simple transformations we obtain

$$
\begin{aligned}
& K_{I I I}\left(a_{k}\right)=\frac{\sqrt{a_{1}} \sqrt{\pi a_{1}} G_{+}}{\sqrt{2 a_{k}}(1+\chi)} \sqrt{\ln \left(\frac{b_{k}}{a_{k}}\right)} X_{k}^{0}(-1, \chi), \\
& K_{I I I}\left(b_{k}\right)=-\frac{\sqrt{a_{1}} \sqrt{\pi a_{1}} G_{+}}{\sqrt{2 b_{k}}(1+\chi)} \sqrt{\ln \left(\frac{b_{k}}{a_{k}}\right)} X_{k}^{0}(1, \chi) \quad\left(\chi=\frac{G_{+}}{G_{-}} ; k=\overline{1, N}\right) .
\end{aligned}
$$

Proceeding from (4.10) we also introduce the infinite SIF:

$$
\begin{aligned}
& K_{I I I}^{0}\left(a_{k}\right)=\frac{K_{I I I}\left(a_{k}\right)}{\sqrt{\pi a_{1}} G_{+}}=\frac{\sqrt{a_{1}}}{\sqrt{2 a_{k}}(1+\chi)} \sqrt{\ln \left(\frac{b_{k}}{a_{k}}\right)} X_{k}^{0}(-1, \chi), \\
& K_{I I I}^{0}\left(b_{k}\right)=\frac{K_{I I I}\left(b_{k}\right)}{\sqrt{\pi a_{1}} G_{+}}=-\frac{\sqrt{a_{1}}}{\sqrt{2 b_{k}}(1+\chi)} \sqrt{\ln \left(\frac{b_{k}}{a_{k}}\right)} X_{k}^{0}(1, \chi) \quad(k=\overline{1, N}) .
\end{aligned}
$$

We will solve values $X_{k}^{0}( \pm 1, x)$ by means of interpolating Lagrange polynomials for functions $X_{k}^{0}(\xi, \chi)$ according to Chebishev's nodes $\eta_{\ell}$ from (3.7). They have the form in [12]:

$$
\begin{aligned}
X_{k}^{0}(1, \chi) & =\frac{1}{M} \sum_{\ell=1}^{M}(-1)^{\ell+1} X_{k}^{0}\left(\eta_{\ell}, \chi\right) \operatorname{ctg}\left(\frac{2 \ell-1}{4 M} \pi\right), \\
X_{k}^{0}(-1, \chi) & =\frac{1}{M} \sum_{\ell=1}^{M}(-1)^{M+1} X_{k}^{0}\left(\eta_{\ell}, \chi\right) \operatorname{tg}\left(\frac{2 \ell-1}{4 M} \pi\right) \quad(k=\overline{1, N}) .
\end{aligned}
$$

Values $X_{k}^{0}( \pm 1, \chi)$ depend on the choice of the natural number $M$, that is, on the number of nodes.

We start solving $J$-integrals. These integrals give the velocities of energy release of the elastic deformation at the end zones of cracks and when the velocities reach a definite critical quantity the cracks are propagated. In the plane problems of piecewise-uniform bodies with cracks it is more convenient to deal with these integrals than with SIF [8].

These integrals are also of interest for piecewise-uniform bodies with cracks at antiplane deformation and therefore here we present the expression of $J$-integral [8]:

$$
J=\frac{1}{4}\left(\frac{1}{G_{+}}+\frac{1}{G_{-}}\right) K_{I I I}^{2}
$$

Furthermore, we consider the relationship $J / J_{0}$ where $J_{0}$ is the corresponding $J$-integral for a homogeneous body made of a material with shear modulus $G_{+}$:

$$
J_{0}=\frac{1}{2 G_{+}} \tilde{K}_{I I}^{2}
$$


Here $\widetilde{K}_{I I I}$ is the SIF in case of a homogeneous body. The quantity $J / J_{0}$ somehow numerically characterizes the possibility of propagation of cracks in piecewise-uniform bodies in comparison with the possibility of propagation in homogeneous bodies.

On the basis of (4.10) we have

$$
\begin{aligned}
& \frac{J\left(a_{k}\right)}{J_{0}\left(a_{k}\right)}=\frac{2}{1+\chi}\left[\frac{X_{k}^{0}(-1, \chi)}{X_{k}^{0}(-1,1)}\right]^{2} \quad\left(\chi=\frac{G_{+}}{G_{-}}\right) \\
& \frac{J\left(b_{k}\right)}{J_{0}\left(b_{k}\right)}=\frac{2}{1+\chi}\left[\frac{X_{k}^{0}(1, \chi)}{X_{k}^{0}(1,1)}\right]^{2} \quad(k=\overline{1, N}) .
\end{aligned}
$$

It is obvious that the relation $J / J_{0}$ is not symmetrical to shear moduli $G_{ \pm}$and to the angles of the wedge openings $\alpha_{ \pm}$, that is, if we substitute $G_{+}$by $G_{-}$and $\alpha_{+}$by $\alpha_{-}$, and as a result the variable components of a piecewise-uniform wedge exchange their places, the value of the relationship will change. To reach the symmetry of $J / J_{0}$ in the mentioned sense unlike [8], we must compare the value of $J$-integral of the composite wedge with the maximum value of $J$-integral of homogeneous wedges with equal angles of the opening $\alpha_{+}+\alpha_{-}$and with the same crack system, manufactured from the material of the upper $\left(G_{+}\right)$or lower $\left(G_{-}\right)$wedges. So we arrive at the function

$$
\begin{gathered}
J\left(\chi, \alpha_{+}, \alpha_{-}\right)=\frac{J}{\max J_{0}}=\frac{1}{4}\left(\frac{1}{G_{+}}+\frac{1}{G_{-}}\right) K_{I I I}^{2} /\left[\frac{1}{2} \max \left(\frac{1}{G_{+}}, \frac{1}{G_{-}}\right) \widetilde{K}_{I I I}^{2}\right]=\frac{K_{I I I}^{2}}{\widetilde{K}_{I I I}^{2}} \delta_{0}^{-1}(\chi), \\
\delta_{0}(\chi)=\left\{\begin{array}{ll}
\frac{2}{1+\chi} & (0<\chi<1) \\
\frac{2 \chi}{1+\chi} & (\chi>1)
\end{array} \quad\left(\chi=\frac{G_{+}}{G_{-}}\right),\right.
\end{gathered}
$$

which as it can be easily seen by means of (4.6) or (4.7) does not change even if the triple $\left(\chi, \alpha_{+}, \alpha_{-}\right)$is substituted by the triple $\left(1 / \chi, \alpha_{-}, \alpha_{+}\right)$, that is, $J\left(\chi, \alpha_{+}, \alpha_{-}\right)=J\left(1 / \chi, \alpha_{-}, \alpha_{+}\right)$. Then instead of (4.15) we have the following characteristics for $J$-integrals:

$$
\begin{aligned}
& J_{a_{k}}\left(\chi, \alpha_{+}, \alpha_{-}\right)=\frac{J\left(a_{k}\right)}{\max J_{0}\left(a_{k}\right)}=\left[\frac{X_{k}^{0}(-1, \chi)}{X_{k}^{0}(-1,1)}\right]^{2} \delta_{0}(\chi), \\
& J_{b_{k}}\left(\chi, \alpha_{+}, \alpha_{-}\right)=\frac{J\left(b_{k}\right)}{\max J_{0}\left(b_{k}\right)}=\left[\frac{X_{k}^{0}(1, \chi)}{X_{k}^{0}(1,1)}\right]^{2} \delta_{0}(\chi) \quad(k=\overline{1, N}) .
\end{aligned}
$$

Finally, the opening of the $k$-th crack $\left(a_{k}, b_{k}\right)$ is determined by the formula

$$
\Phi_{k}(r)=\int_{a_{k}}^{r} \varphi\left(r_{0}\right) d r_{0} \quad\left(a_{k} \leq r \leq b_{k} ; k=\overline{1, N}\right),
$$


which by virtue of (3.1), (3.4), and (3.9) is transformed into the form

$$
\Phi_{k}^{0}(\xi)=\ln \sqrt{\frac{b_{k}}{a_{k}}} \int_{-1}^{\xi} \frac{X_{k}^{0}(\eta, \chi)}{\sqrt{1-\eta^{2}}} d \eta \quad(-1 \leq \xi \leq 1 ; k=\overline{1, N})
$$

and $\Phi_{k}^{0}( \pm 1)=0$. Here an infinite crack opening is introduced:

$$
\Phi_{k}^{0}(\xi)=a_{1}^{-1} \Phi_{k}\left[a_{1} \exp \left(\frac{\beta_{k}-\alpha_{k}}{2} \xi+\frac{\beta_{k}+\alpha_{k}}{2}\right)\right] \quad(k=\overline{1, N}) .
$$

By applying the corresponding quadrature formula we have

$$
\Phi_{k}^{0}(\xi)=\frac{\pi}{M} \ln \sqrt{\frac{b_{k}}{a_{k}}} \sum_{\eta_{\ell} \leq \xi} X_{k}^{0}\left(\eta_{\ell}, \chi\right) \quad(k=\overline{1, N}) .
$$

Hence

$$
\Phi_{k}^{0}\left(\xi_{n}\right)=\frac{\pi}{M} \ln \sqrt{\frac{b_{k}}{a_{k}}} \sum_{\eta_{\ell} \leq \xi_{n}} X_{k}^{0}\left(\eta_{\ell}, \chi\right) \quad(n=\overline{1, M-1}) .
$$

Thus, an infinite opening of the $k$-th crack may be solved by (4.21) or by (4.22).

\section{Numerical results and conclusions}

For numerical realization of the problem being investigated here, firstly, it is necessary to choose natural numbers $M, N$ and at the given values of the physical parameter $\chi(\chi=$ $G_{+} / G_{-}$) the geometric parameters

$$
\alpha_{+}, \alpha_{-}, \quad c_{k}=\frac{a_{k}}{a_{1}}, \quad d_{k}=\frac{b_{k}}{a_{1}}\left(c_{1}=1, k=\overline{1, N}\right),
$$

and also at the given loads $\tau_{ \pm}^{0}(t), f_{ \pm}^{0}(t)$ (see formulas (3.3e) and (3.3f)) to calculate the quantities included into the algebraic system of the formulas (3.10). After solving this system, the SIF is calculated by formulas (4.11), J-integrals by formulas (4.15) or (4.17) and crack openings by the formula (4.22). And values $X_{k}^{0}( \pm 1, x)(k=\overline{1, N})$ are calculated by (4.12).

The described procedure may be illustrated in two cases. 


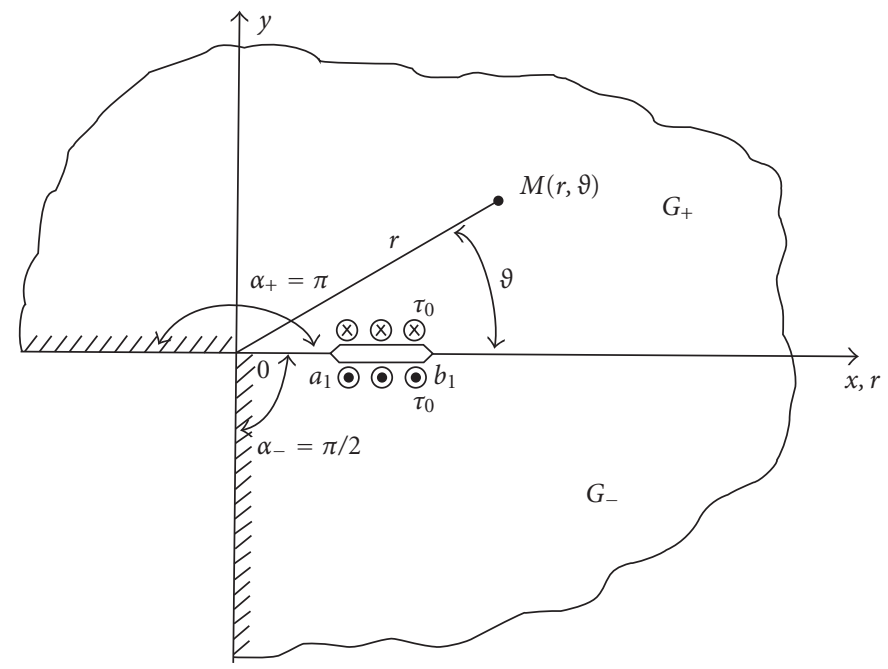

Figure 5.1. An antiplane deformation of the piecewise-uniform wedge consisting of a half-plane and quarter-plane, and containing a single inner crack $L=\left(a_{1}, b_{1}\right)$ at the interface of media, the edges of which are uniformly loaded by the distributed forces $\tau_{0}$.

Case I. Let $N=1, \alpha_{+}=\pi, \alpha_{-}=\pi / 2, f_{+}(r)=f_{-}(r) \equiv 0, \tau_{+}(r)=\tau_{-}(r) \equiv \tau_{0}=$ const (Figure 5.1).

In the given case by (5.1),

$$
\gamma=\frac{\pi}{2} ; \quad c_{1}=1 ; \quad d_{1}=\frac{b_{1}}{a_{1}}
$$

by $(2.29 \mathrm{c}),(3.2 \mathrm{c}),(3.2 \mathrm{~d}),(3.5 \mathrm{~d})$, and (3.5f),

$$
\psi_{K}\left(\chi, \alpha_{+}, \alpha_{-}\right)=\frac{\chi}{\chi+1} ; \quad \nu_{1}=\frac{1}{2} \ln d_{1} ; \quad \delta_{11}=1 ; \quad \gamma_{1}=\frac{\pi}{2 \ln d_{1}}
$$

and by (2.27b), (2.30c), (3.3b), (3.3i), and (3.5c),

$$
\begin{aligned}
& \tilde{Q}_{0}(t)= \int_{0}^{\infty}\left\{\frac{\chi \operatorname{th}(\pi \lambda)[1-\operatorname{th}(\pi \lambda / 2)]+\operatorname{th}(\pi \lambda / 2)[1-\operatorname{th}(\pi \lambda)]}{\chi \operatorname{th}(\pi \lambda)+\operatorname{th}(\pi \lambda / 2)}-\frac{2 \chi}{1+\chi} e^{-\pi \lambda}\right\} \\
& \times \sin (\lambda t) d \lambda \quad(-\infty<t<\infty) ; \\
& F_{1}^{0}(\xi)=-(1+\chi) \bar{\tau}_{0} d_{1}^{(1+\xi) / 2} \quad\left(\bar{\tau}_{0}=\frac{\tau_{0}}{G_{+}}\right) .
\end{aligned}
$$

Further, proceeding from $(5.4 \mathrm{~b})$, the solution of system $(3.10)$ in the first part, equal to $G_{1}^{0}\left(\xi_{n}\right)(n=1, M-1)$, where

$$
G_{1}^{0}(\xi)=-d_{1}^{(1+\xi) / 2} \quad(-1<\xi<1)
$$


is designated with $\omega_{1}^{0}\left(\eta_{\ell}, \chi\right)$. Then

$$
X_{1}^{0}\left(\eta_{\ell}, \chi\right)=(1+\chi) \bar{\tau}_{0} \omega_{1}^{0}\left(\eta_{\ell}, \chi\right) \quad(\ell=\overline{1, M})
$$

To investigate the behavior of SIF $K_{a_{1}}^{0}$ at approaching the left crack tip $r=a_{1}$ to the tip of the composite wedge $O$ in the investigated case it is convenient instead of (4.11) to introduce the following infinite SIF:

$$
\begin{aligned}
& \tilde{K}_{I I I}^{0}\left(a_{1}\right)=\frac{K_{I I I}\left(a_{1}\right)}{\sqrt{\pi b_{1}} \tau_{0}}=\frac{1}{\sqrt{2 d_{1}}} \sqrt{\ln d_{1}} \omega_{1}^{0}(-1, \chi), \\
& \tilde{K}_{I I I}^{0}\left(b_{1}\right)=\frac{K_{I I I}\left(b_{1}\right)}{\sqrt{\pi b_{1}} \tau_{0}}=-\frac{1}{d_{1} \sqrt{2}} \sqrt{\ln d_{1}} \omega_{1}^{0}(1, \chi) .
\end{aligned}
$$

Values $\omega_{1}^{0}( \pm 1, \chi)$ are again defined by formulas (4.12) in which quantities $X_{1}^{0}\left(\eta_{\ell}, \chi\right)$ must be substituted by quantities $\omega_{1}^{0}\left(\eta_{\ell}, \chi\right)$.

In the given case the quantities of the relations of $J$-integrals in correspondence with (4.17) and (5.6) will be expressed by the formulas

$$
\begin{aligned}
& J_{a_{1}}\left(\chi, \alpha_{+}, \alpha_{-}\right)=\frac{J\left(a_{1}\right)}{\max J_{0}\left(a_{1}\right)}=\delta_{0}(\chi)\left[\frac{\omega_{1}^{0}(-1, \chi)}{\omega_{1}^{0}(-1,1)}\right]^{2}, \\
& J_{b_{1}}\left(\chi, \alpha_{+}, \alpha_{-}\right)=\frac{J\left(b_{1}\right)}{\max J_{0}\left(b_{1}\right)}=\delta_{0}(\chi)\left[\frac{\omega_{1}^{0}(1, \chi)}{\omega_{1}^{0}(1,1)}\right]^{2}
\end{aligned}
$$

Finally, according to (4.22) for a crack opening we will have

$$
\tilde{\Phi}_{k}^{0}\left(\xi_{n}\right)=\frac{\pi}{M} \ln \sqrt{d_{1}}(1+\chi) \sum_{\eta_{\ell} \leq \xi_{n}} \omega_{1}^{0}\left(\eta_{\ell}, \chi\right),
$$

where

$$
\widetilde{\Phi}_{k}^{0}(\xi)=\frac{G_{+}}{\tau_{0}} \Phi_{k}^{0}(\xi)=\frac{1}{\bar{\tau}_{0}} \Phi_{k}^{0}(\xi) \quad(-1 \leq \xi \leq 1)
$$

Thus, in the considered case, systems (3.10) and formulas (5.4)-(5.10) will be design formulas.

Case II. Let now $N=2$ and again $\alpha_{+}=\pi, \alpha_{-}=\pi / 2, f_{+}(r)=f_{-}(r)=0$ (Figure 5.2). As to loading crack edges here we must consider the following three underloading operations of their edges:

(1) the crack edges $\left(a_{1}, b_{1}\right)$ are loaded by the same uniformly distributed intensity forces $\tau_{0}$, but the crack edges $\left(a_{2}, b_{2}\right)$ are free of loads;

(2) the crack edges $\left(a_{2}, b_{2}\right)$ are loaded by the same uniformly distributed forces $\tau_{0}$, but the crack edges $\left(a_{1}, b_{1}\right)$ are free of forces;

(3) the crack edges $\left(a_{1}, b_{1}\right)$ and $\left(a_{2}, b_{2}\right)$ are loaded simultaneously by uniformly distributed forces $\tau_{0}$. 


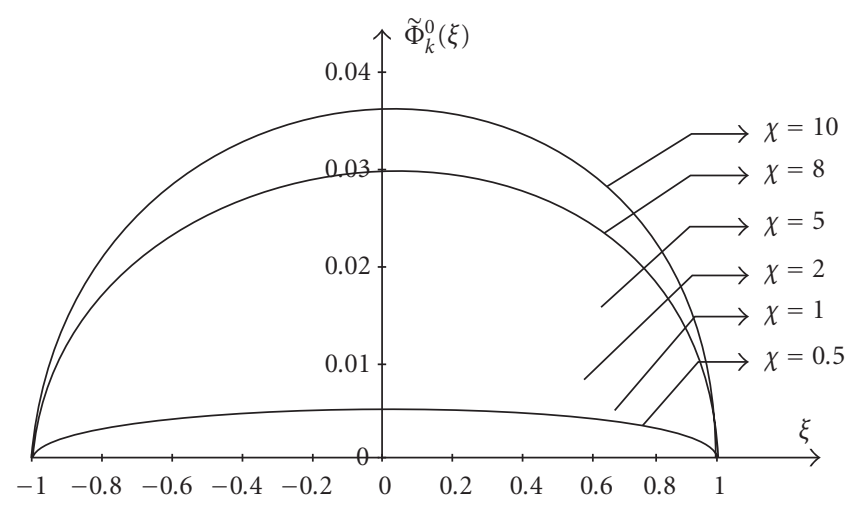

Figure 5.2. Changes of infinite crack openings $\widetilde{\Phi}_{k}^{0}(\xi)$ calculated by the formulas (5.9)-(5.10) in the interval $1 \leq \xi \leq 1$ at $d_{1}=1,2$ and at different values of the parameter $\chi$.

In this case in (5.1),

$$
\gamma=\frac{\pi}{2} ; \quad c_{1}=1, \quad c_{2}=\frac{a_{2}}{a_{1}} ; \quad d_{1}=\frac{b_{1}}{a_{1}}, \quad d_{2}=\frac{b_{2}}{a_{1}} ;
$$

in $(2.29 \mathrm{c}),(3.2 \mathrm{c}),(3.2 \mathrm{~d}),(3.5 \mathrm{~d})$, and $(3.5 \mathrm{f})$,

$$
\begin{gathered}
\psi_{K}\left(\chi, \alpha_{+}, \alpha_{-}\right)=\frac{\chi}{\chi+1} ; \quad \alpha_{1}=0, \quad \beta_{1}=\ln d_{1} ; \\
\alpha_{2}=\ln c_{2}, \quad \beta_{2}=\ln d_{2}, \quad \nu_{1}=\frac{1}{2} \ln d_{1}, \quad \nu_{2}=\frac{1}{2} \ln \frac{d_{2}}{c_{2}}=\frac{1}{2} \ln \frac{b_{2}}{a_{2}} ; \\
\delta_{12}=\frac{\ln d_{1}}{\ln \left(d_{2} / c_{2}\right)}, \quad \delta_{21}=\delta_{12}^{-1}=\frac{\ln \left(d_{2} / c_{2}\right)}{\ln d_{1}} \\
\delta_{11}=\delta_{22}=1 ; \quad \gamma_{1}=\frac{\pi}{2 \ln d_{1}}, \quad \gamma_{2}=\frac{\pi}{2 \ln \left(d_{2} / c_{2}\right)}
\end{gathered}
$$

and in (3.3b), (3.3c), (3.3d), (3.3e), (3.3f), (3.3g), (3.3h), (3.3i), (3.5d), (3.5e), and (3.5f),

$$
\begin{aligned}
& F_{1}^{0}(\xi)=-(1+\chi) \bar{\tau}_{0} d_{1}^{(1+\xi) / 2}, \\
& F_{2}^{0}(\xi)=-(1+\chi) \bar{\tau}_{0} c_{2}^{(1-\xi) / 2} d_{2}^{(1+\xi) / 2} \quad\left(\bar{\tau}=\frac{\tau_{0}}{G_{+}}\right) .
\end{aligned}
$$

It must be noted here that in the given case we will have again the kernel function $\widetilde{Q}_{0}(t)$ from (5.4a).

Further, we introduce the functions

$$
\begin{aligned}
& G_{1}^{0}(\xi)=-d_{1}^{(1+\xi) / 2} \\
& G_{2}^{0}(\xi)=-c_{2}^{(1-\xi) / 2} d_{2}^{(1+\xi) / 2} \quad(-1<\xi<1)
\end{aligned}
$$


and the solution of the system (3.10) at the right parts equal to

$$
\left\{G_{1}^{0}\left(\xi_{n}\right), 0\right\} \quad(n=\overline{1, M-1}) .
$$

We denote

$$
\left\{\omega_{1}^{01}\left(\eta_{\ell}, \chi\right), \omega_{2}^{01}\left(\eta_{\ell}, \chi\right)\right\} \quad(\ell=\overline{1, M}),
$$

but the solution of this system at right parts is equal to

$$
\left\{0, G_{2}^{0}\left(\xi_{n}\right)\right\} \quad(n=\overline{1, M-1}) .
$$

We denote

$$
\left\{\omega_{1}^{02}\left(\eta_{\ell}, \chi\right), \omega_{2}^{02}\left(\eta_{\ell}, \chi\right)\right\} \quad(\ell=\overline{1, M}) .
$$

Then, at the first, second, and third loading operations of the crack edges, quantities $X_{k}^{0}\left(\eta_{\ell}, \chi\right)$ will have the same form, respectively:

$$
\begin{aligned}
& X_{k}^{0}\left(\eta_{\ell}, \chi\right)=(1+\chi) \bar{\tau}_{0} \omega_{k}^{01}\left(\eta_{\ell}, \chi\right), \\
& X_{k}^{0}\left(\eta_{\ell}, \chi\right)=(1+\chi) \bar{\tau}_{0} \omega_{k}^{02}\left(\eta_{\ell}, \chi\right), \\
& X_{k}^{0}\left(\eta_{\ell}, \chi\right)=(1+\chi) \bar{\tau}_{0}\left[\omega_{k}^{01}\left(\eta_{\ell}, \chi\right)+\omega_{k}^{02}\left(\eta_{\ell}, \chi\right)\right] \quad(k=1,2) .
\end{aligned}
$$

Furthermore, with the help of (5.19) we write the expressions of SIF (4.11) that may be used in the given case according to the mentioned loading operations of the crack edges:

$$
\begin{aligned}
& K_{I I I}^{0 j}\left(a_{1}\right)=\frac{K_{I I I}^{j}\left(a_{1}\right)}{\sqrt{\pi a_{1}} \tau_{0}}=\sqrt{\frac{1}{2} \ln d_{1}} \omega_{1}^{0 j}(-1, \chi), \\
& K_{I I I}^{0 j}\left(b_{1}\right)=\frac{K_{I I I}^{j}\left(b_{1}\right)}{\sqrt{\pi a_{1}} \tau_{0}}=-\frac{1}{\sqrt{d_{1}}} \sqrt{\frac{1}{2} \ln d_{1}} \omega_{1}^{0 j}(1, \chi), \\
& K_{I I I}^{0 j}\left(a_{2}\right)=\frac{K_{I I I}^{j}\left(a_{2}\right)}{\sqrt{\pi a_{1}} \tau_{0}}=\frac{1}{\sqrt{c_{2}}} \sqrt{\frac{1}{2} \ln \frac{d_{2}}{c_{2}}} \omega_{2}^{0 j}(-1, \chi), \\
& K_{I I I}^{0 j}\left(b_{2}\right)=\frac{K_{I I I}^{j}\left(b_{2}\right)}{\sqrt{\pi a_{1}} \tau_{0}}=-\frac{1}{\sqrt{d_{2}}} \sqrt{\frac{1}{2} \ln \frac{d_{2}}{c_{2}}} \omega_{2}^{0 j}(1, \chi) \quad(j=1,2) .
\end{aligned}
$$

It should be mentioned here that at $j=1$ we have the first loading operation, at $j=2$ the second one, and at the third loading operation according to (5.19c) the SIF becomes a simple linear superposition of the corresponding SIF at the first and second operations. 
266 Stress deformation condition of a piecewise-uniform wedge

Table 5.1. $\widetilde{K}_{I I I}^{0}\left(a_{1}\right)$ and $\widetilde{K}_{I I I}^{0}\left(b_{1}\right)$ values.

\begin{tabular}{|c|c|c|c|c|c|c|c|}
\hline \multicolumn{8}{|c|}{$d_{1}$} \\
\hline$\chi$ & 1.2 & 1.5 & 2 & 4 & 8 & 10 & 20 \\
\hline \multirow{2}{*}{0.1} & 0.00962832 & 0.0136512 & 0.0168313 & 0.0213551 & 0.0246976 & 0.0258072 & 0.0298368 \\
\hline & 0.00962782 & 0.0136434 & 0.0167832 & 0.0208872 & 0.0230058 & 0.0234829 & 0.0245993 \\
\hline \multirow{2}{*}{0.3} & 0.00962917 & 0.0136575 & 0.0168548 & 0.0214822 & 0.0250238 & 0.0262172 & 0.0305629 \\
\hline & 0.00962859 & 0.0136484 & 0.0167999 & 0.0209526 & 0.0231318 & 0.0236283 & 0.0248006 \\
\hline \multirow{2}{*}{0.5} & 0.00962975 & 0.0136617 & 0.016871 & 0.0215697 & 0.0252486 & 0.0264997 & 0.0310631 \\
\hline & 0.00962912 & 0.0136519 & 0.0168114 & 0.0209977 & 0.0232189 & 0.0237288 & 0.0249402 \\
\hline \multirow{2}{*}{1} & 0.00963064 & 0.0136682 & 0.0168956 & 0.0217031 & 0.0255916 & 0.0269308 & 0.0318268 \\
\hline & 0.00962993 & 0.0136573 & 0.0168289 & 0.0210666 & 0.0233522 & 0.0238828 & 0.0251544 \\
\hline \multirow{2}{*}{2} & 0.00963146 & 0.0136743 & 0.0169186 & 0.0218274 & 0.0259112 & 0.0273327 & 0.0325386 \\
\hline & 0.00963068 & 0.0136622 & 0.0168452 & 0.0211309 & 0.0234767 & 0.024027 & 0.0253555 \\
\hline \multirow{2}{*}{5} & 0.00963223 & 0.01368 & 0.01694 & 0.0219439 & 0.0262111 & 0.0277097 & 0.0332067 \\
\hline & 0.00963138 & 0.0136668 & 0.0168604 & 0.0211911 & 0.023594 & 0.0241627 & 0.0255453 \\
\hline \multirow{2}{*}{8} & 0.00963248 & 0.0136818 & 0.0169469 & 0.0219812 & 0.0263071 & 0.0278305 & 0.0334207 \\
\hline & 0.00963161 & 0.0136683 & 0.0168653 & 0.0212105 & 0.0236316 & 0.0242063 & 0.0256064 \\
\hline \multirow{2}{*}{10} & 0.00963257 & 0.0136824 & 0.0169494 & 0.0219945 & 0.0263416 & 0.0278738 & 0.0334976 \\
\hline & 0.00963169 & 0.0136688 & 0.0168671 & 0.0212174 & 0.0236451 & 0.0242219 & 0.0256283 \\
\hline
\end{tabular}

By analogy with (4.17), (5.19a), and (5.19b) for relations of $J$-integrals we have

$$
\begin{aligned}
& \frac{J^{j}\left(a_{1}\right)}{\max J_{0}^{j}\left(a_{1}\right)}=J_{a_{1}}^{j}\left(\chi, \alpha_{+}, \alpha_{-}\right)=\delta_{0}(\chi)\left[\frac{\omega_{1}^{0 j}(-1, \chi)}{\omega_{1}^{0 j}(-1,1)}\right]^{2}, \\
& \frac{J^{j}\left(b_{1}\right)}{\max J_{0}^{j}\left(b_{1}\right)}=J_{b_{1}}^{j}\left(\chi, \alpha_{+}, \alpha_{-}\right)=\delta_{0}(\chi)\left[\frac{\omega_{1}^{0 j}(1, \chi)}{\omega_{1}^{0 j}(1,1)}\right]^{2}, \\
& \frac{J^{j}\left(a_{2}\right)}{\max J_{0}^{j}\left(a_{2}\right)}=J_{a_{2}}^{j}\left(\chi, \alpha_{+}, \alpha_{-}\right)=\delta_{0}(\chi)\left[\frac{\omega_{2}^{0 j}(-1, \chi)}{\omega_{2}^{0 j}(-1,1)}\right]^{2}, \\
& \frac{J^{j}\left(b_{2}\right)}{\max J_{0}^{j}\left(b_{2}\right)}=J_{b_{2}}^{j}\left(\chi, \alpha_{+}, \alpha_{-}\right)=\delta_{0}(\chi)\left[\frac{\omega_{2}^{0 j}(1, \chi)}{\omega_{2}^{0 j}(1,1)}\right]^{2} \quad(j=1,2) .
\end{aligned}
$$

Here we can write easily the following formulas for solving the crack openings.

Thus, in the discussed case the design formulas will be the systems (3.10), (5.4a) and the formulas (5.13)-(5.21).

Further we give the numerical results only in the first case, introducing

$$
\chi=\frac{G_{+}}{G_{-}}=0.1 ; 0.3 ; 0.5 ; 1,2 ; 5 ; 8 ; 10, \quad d_{1}=1.2 ; 1.5 ; 2 ; 4 ; 8 ; 10 ; 20 .
$$

In Table 5.1 the formulas calculated by (5.7), values of infinite SIF $\widetilde{K}_{I I I}^{0}\left(a_{1}\right)$, and $\widetilde{K}_{I I I}^{0}\left(b_{1}\right)$ at different values of the parameters $d_{1}$ and $\chi$ are given, and in each square of Table 5.1 
Table 5.2. $J_{a_{1}}\left(\chi, \alpha_{+}, \alpha_{-}\right)$and $J_{b_{1}}\left(\chi, \alpha_{+}, \alpha_{-}\right)$values.

\begin{tabular}{cccccccc}
\hline & \multicolumn{7}{c}{$d_{1}$} \\
\hline$\chi$ & 1.2 & 1.5 & 2 & 4 & 8 & 10 & 20 \\
\hline \multirow{2}{*}{0.1} & 1.81731 & 1.81366 & 1.80436 & 1.76033 & 1.69337 & 1.66962 & 1.59793 \\
& 1.81738 & 1.81448 & 1.80832 & 1.78734 & 1.76465 & 1.7578 & 1.73881 \\
\hline \multirow{2}{*}{0.3} & 1.53799 & 1.53603 & 1.53104 & 1.5073 & 1.47096 & 1.45801 & 1.4187 \\
& 1.53803 & 1.53648 & 1.53316 & 1.52185 & 1.50957 & 1.50584 & 1.49548 \\
\hline \multirow{2}{*}{0.5} & 1.33309 & 1.33206 & 1.32945 & 1.31699 & 1.29783 & 1.29098 & 1.27012 \\
& 1.33311 & 1.33229 & 1.33056 & 1.32463 & 1.31816 & 1.31619 & 1.31071 \\
\hline \multirow{2}{*}{1} & 1.0 & 1.0 & 1.0 & 1.0 & 1.0 & 1.0 & 1.0 \\
& 1.0 & 1.0 & 1.0 & 1.0 & 1.0 & 1.0 & 1.0 \\
\hline \multirow{2}{*}{2} & 1.33356 & 1.33452 & 1.33696 & 1.34864 & 1.36685 & 1.37342 & 1.39364 \\
& 1.33354 & 1.3343 & 1.33592 & 1.34148 & 1.3476 & 1.34947 & 1.35473 \\
\hline \multirow{2}{*}{5} & 1.66722 & 1.66953 & 1.67544 & 1.70385 & 1.74833 & 1.76446 & 1.81432 \\
& 1.66717 & 1.66901 & 1.67292 & 1.68643 & 1.70136 & 1.70595 & 1.71887 \\
\hline \multirow{2}{*}{8} & 1.77846 & 1.7813 & 1.78859 & 1.82362 & 1.87858 & 1.89854 & 1.96031 \\
& 1.7784 & 1.78066 & 1.78548 & 1.80214 & 1.82058 & 1.82626 & 1.84223 \\
\hline \multirow{2}{*}{10} & 1.81891 & 1.82196 & 1.82977 & 1.86734 & 1.92631 & 1.94774 & 2.01409 \\
& 1.81885 & 1.82127 & 1.82644 & 1.8443 & 1.86409 & 1.87018 & 1.88733 \\
\hline
\end{tabular}

in the first lines values $\tilde{K}_{I I I}^{0}\left(a_{1}\right)$ are written, and in the second lines the values $\widetilde{K}_{I I I}^{0}\left(b_{1}\right)$ are written. The analysis of these data shows that at a fixed value of $d_{1}$ the values of SIF change insignificantly when $\chi$ is changed. In this case, the nearest to the wedge tip SIF $\widetilde{K}_{I I I}^{0}\left(a_{1}\right)$ is larger than SIF $\widetilde{K}_{I I I}^{0}\left(b_{1}\right)$. When $\chi$ is fixed the infinite SIF of the parameter $d_{1}$ which may be considered to be the approach of the crack tip $a_{1}$ to the wedge tip increases.

In Table 5.2 the values of $J$-integrals calculated by the formulas (5.8) at different values of the parameters $d_{1}$ and $\chi$ are given, and in Table 5.1, in the first lines values $J_{a_{1}}\left(\chi, \alpha_{+}, \alpha_{-}\right)$ are given, and in the second lines values $J_{b_{1}}\left(\chi, \alpha_{+}, \alpha_{-}\right)$are given. The analysis of these data shows that heterogeneity (values $\chi$ ) of the wedge at fixed $d_{1}$ significantly influences the quantities of $J$-integrals. It is easy to discover that at $0<\chi<1$ the values of $J$-integrals decrease but at $\chi>1$ increase.

In Figure 5.2 the way of changes of infinite crack openings $\widetilde{\Phi}_{k}^{0}(\xi)$ calculated by formulas (5.9)-(5.10) in the interval $-1 \leq \xi \leq 1$ at $d_{1}=1,2$ and at different values of the parameter $\chi$ is shown. The graphs $\widetilde{\Phi}_{k}^{0}(\xi)$ at other values of the parameters $d_{1}$ and $\chi$ have the same form.

\section{References}

[1] G. Beйtmen, A. Èrdeĭi, V. Magnus, F. Oberhettinger, and F. Trikomi, Tables of Integral Transforms. Vol. I. Fourier, Laplace, Mellin transforms, Izdat. Nauka, Moscow, 1969.

[2] B. A. Bilby and J. D. Eshelby, Dislocation and the theory of fracture, Fracture, An Advanced Treatise, Academic Press, New York, 1968, pp. 99-182.

[3] G.P. Cherepanov, Mechanics of Brittle Fracture, McGraw-Hill International Book Company, 1979. 
[4] F. Erdogan and G. D. Gupta, Bonded wedges with an interface crack under anti-plane shear loading, J. Fract. Mech 11 (1975), no. 4, 583-597.

[5] F. Erdogan, G. D. Gupta, and T. S. Cook, Numerical solution of singular integral equations, Mechanics of Fracture, vol. 1, Noordhoff International Publishing, Leiden, 1973, pp. 368-425.

[6] G. Kuhn, Inhomogen geschichteten Keil mit Riss unter nichtebenen Schubspannungszustand, Z. Angew. Math. Mech. 56 (1976), no. 3, 125-127 (German).

[7] M. J. Lighthill, Introduction to Fourier Analysis and Generalised Functions, Cambridge University Press, New York, 1960.

[8] Y. Marakami (ed.), Stress Intensity Factors Handbook, vol. 1, Pergamon Press, 1987.

[9] M. C. Mkrtichian and S. M. Mkhitarian, On the problem of a stressed state complex elastic infinite body with a periodical system of collinear cracks at a longitudinal shear, Proc. Natl. Acad. Sci. USA 94 (1993), no. 2, 104-109.

[10] N. I. Muskhelishvili, Singular Integral Equations, Wolters-Noordhoff Publishing, Groningen, 1958.

[11] V. V. Panasyuk (ed.), Fracture Mechanics and Strength of Materials, vol. 2, Naukova-Dumka, Kiev, 1988, A Handbook.

[12] V. V. Panasyuk, M. P. Savruk, and A. P. Datsyshin, Distribution of Stresses Near Cracks in Plates and Shells, Izdat. Naukova-Dumka, Kiev, 1976.

[13] M. P. Savruk, Stress Intensity Factors in Cracked Bodies, Naukova-Dumka, Kiev, 1988.

[14] P. S. Theocaris and N. I. Ioakimidis, Numerical integration methods for the solution of singular integral equations, Quart. Appl. Math. 35 (1977/78), no. 1, 173-187.

[15] P. S. Theocaris and G. Makrakis, Crack kinking in anti-plane shear solved by the Mellin transform, Internat. J. Fracture 34 (1987), no. 4, 251-262.

[16] J. S. Ufliand, Integral Transformations in the Problems of the Theory of Elasticity, Nauka, Leningrad, 1968.

D. I. Bardzokas: Laboratory of Strength and Materials, Department of Mechanics, Faculty of Applied Sciences, National Technical University of Athens, Zografou Campus, Theocaris Building, 15773 Athens, Greece

E-mail address: bardim@central.ntua.gr

S. H. Gevorgyan: State Engineering University of Armenia, 105 Terian Street, 375009 Yerevan, Armenia

S. M. Mkhitaryan: Institute of Mechanics, National Academy of Sciences of Armenia, 24 Marshall Baghramian Avenue, 375019 Yerevan, Armenia 


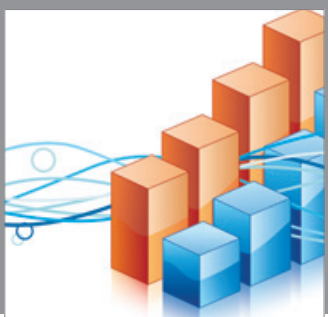

Advances in

Operations Research

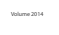

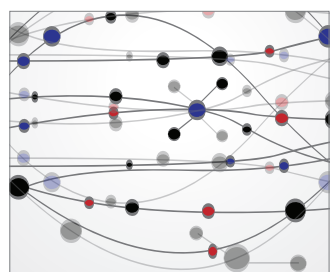

\section{The Scientific} World Journal
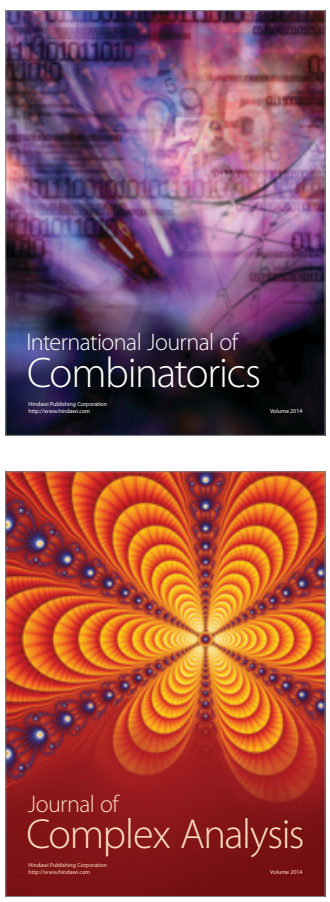

International Journal of

Mathematics and

Mathematical

Sciences
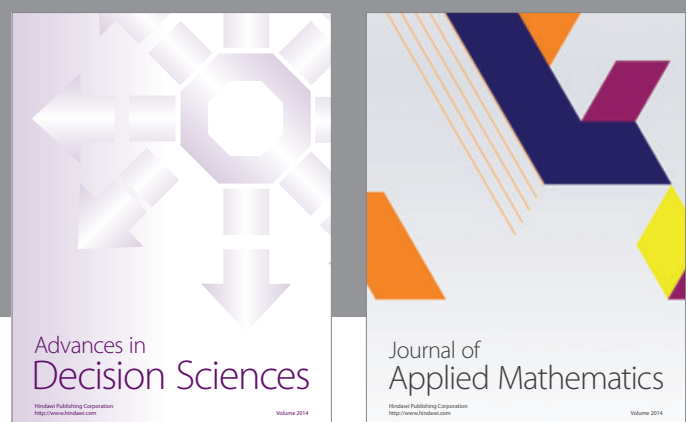

Journal of

Applied Mathematics
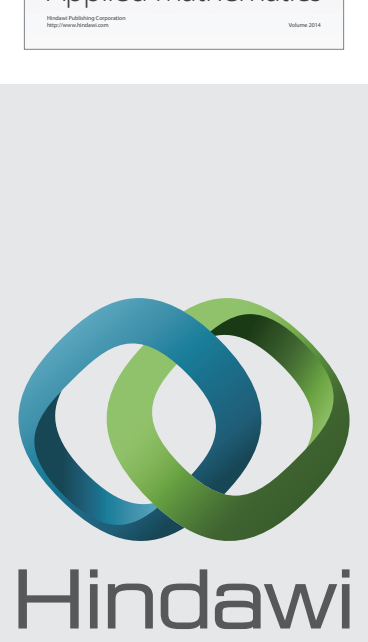

Submit your manuscripts at http://www.hindawi.com
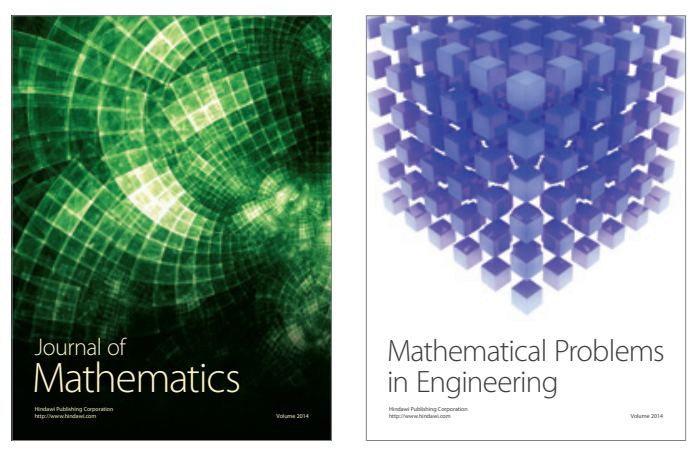

Mathematical Problems in Engineering
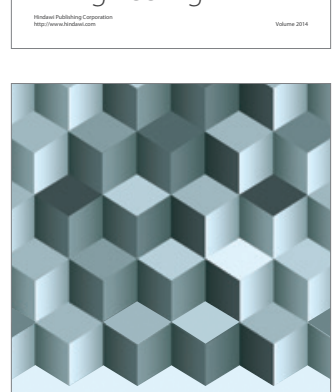

Journal of

Function Spaces
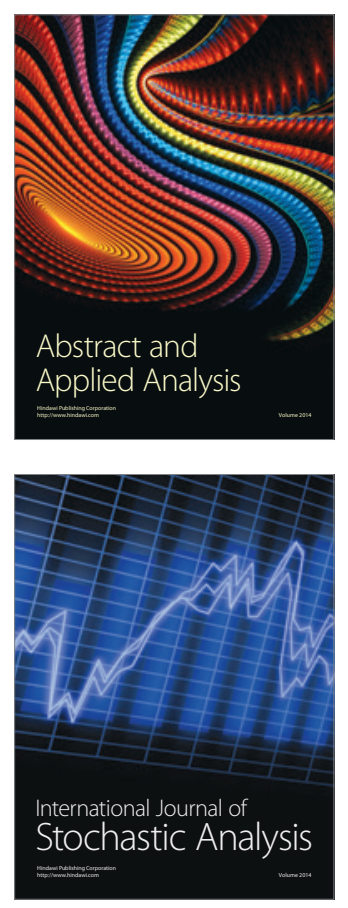

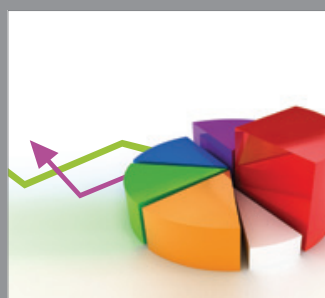

ournal of

Probability and Statistics

Promensencen
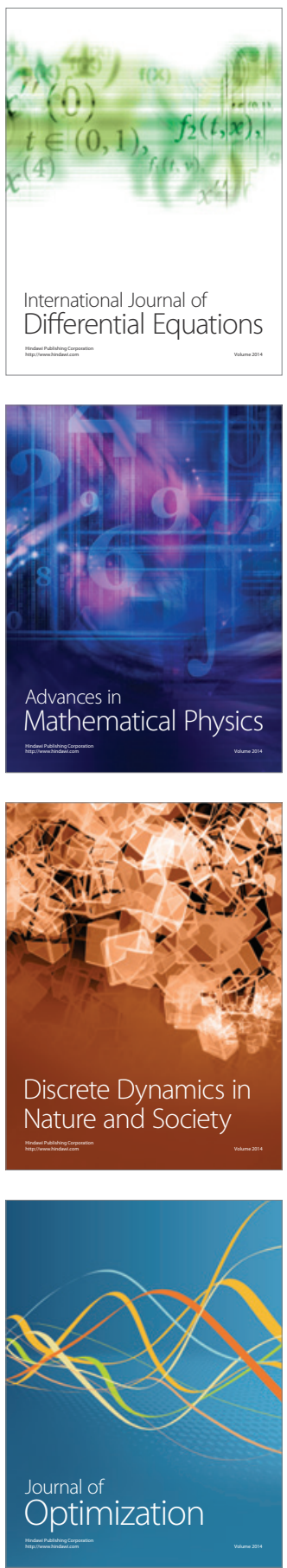